\title{
Incidence of neonatal sepsis in relation to prolonged rupture of membranes (PROM) $>18$ hours and associated risk factors for early onset neonatal sepsis (EONS)
}

\author{
Chakravarthi G.K. ${ }^{1}$, S. Surya Veera Kumar ${ }^{2}$ \\ ${ }^{1}$ Dr. G. Kalyan Chakravarthi, Associate Professor, ${ }^{2}$ Dr. S. Surya Veera Kumar, Assistant Professor; both authors are \\ attached with Department of Paediatrics, GSL Medical College \& General Hospital, Rajahmundry (AP).
}

Corresponding Author: Dr. S. Surya Veera Kumar, Assistant Professor, Department of Pediatrics, GSL Medical College \& General Hospital, Rajahmundry (AP) India. E-mail: siddu211@gmail.com

\begin{abstract}
Introduction: PROM is significant not only in perinatal morbidity and mortality, but also in the long-term neonatal complications and sequelae in survived neonates. The evaluation of neonatal sepsis is important so as to institute treatment as quickly as possible. Hence the present study is undertaken to determine the incidence of early onset neonatal sepsis in relation to PROM of more than 18 hours. Method: The present prospective study was conducted from December 2013 to November 2014 in GSL medical college and hospital, Rajahmundry. All the neonates born to mothers with history of prolonged rupture of membranes $>18 \mathrm{hrs}$ during study period was formed sample size for present study. Institutional Ethical Clearance was obtained prior begin of the study. A detailed history was taken including age, parity, Obstetric history of the mother with emphasis on exact time of rupture of membranes, duration history and antibiotics before labour were evaluated. Result: The incidence of EONS in present study was found to be $14.5 \%$. In present study the incidence of sepsis is higher in low birth weight neonates (66\%) compared to normal birth weight babies (34\%) and higher rate of incidence in preterm neonates (61.5\%) than in term neonates (38.5\%). Further, Staphylococcus aureus was the commonest isolate (45.45\%) followed by CONS (27.27\%). Conclusion: In conclusion, evaluation of neonatal sepsis is very important so as to institute treatment as quickly as possible.
\end{abstract}

Keywords: Neonatal sepsis, Prolonged rupture of membranes (PROM), Early Onset Neonatal Sepsis (EONS), Bacterial pathogens

\section{Introduction}

Prolonged rupture of membrane (PROM) is an important risk factor for early Onset neonatal sepsis (EONS), which is associated with increased neonatal morbidity and mortality. This study is mainly done to know the incidence of early onset neonatal sepsis and associated risk factors for early onset neonatal sepsis in neonates born to mothers with history of PROM.

According to the World Health Organization, approximately four million neonates die annually with a global neonatal mortality rate of 23/1,000 live births $[1,2]$. About a million of these deaths are attributable to

Manuscript received: $30^{\text {th }}$ July 2019

Reviewed: $7^{\text {th }}$ August 2019

Author Corrected: $14^{\text {th }}$ August 2019

Accepted for Publication: $17^{\text {th }}$ August 2019 neonatal infections [3]. The incidence of neonatal sepsis according to the data from National Neonatal Perinatal Data base (NNPD 2002-03) is 30 per 1000 live births. It was reported as high as three times this number in developing countries compared to developed countries [4]. Prolonged rupture of membrane (PROM), defined as rupture of membrane lasting more than 18 hours before labor, is found in approximately $8 \%-10 \%$ of all pregnancies $[5,6]$.

PROM is an important risk factor for both early onset neonatal sepsis (EONS)and preterm births [7-9]. Blood culture is the most frequently asked microbiological investigations for sick neonates. It is the gold standard for diagnosis of septicemia and should be performed in

Pediatric Review: International Journal of Pediatric Research Available online at: www.medresearch.in 444|P a g e 


\section{Original Research Article}

all cases of suspected sepsis prior to starting antibiotics. A positive blood culture with sensitivity of isolated organism is the best guide to antimicrobial therapy. In EOS urine culture has a low yield and is not indicated.

Urine cultures obtained by suprapubic puncture or bladder catheterization have been recommended in all cases of late onset sepsis. Neonatal sepsis is usually associated with leucopenia $<5000 \mathrm{cu} / \mathrm{cumm}$. This finding was taken along with other parameters for early diagnosis of neonatal sepsis with reasonable sensitivity and specificity.

PROM is significant not only in perinatal morbidity and mortality, but also in the long-term neonatal complications and sequelae in survived neonates [10]. Improved prenatal care and antenatal antimicrobial treatment of women with a history of PROM had significantly improved neonatal outcome in association with early detection of sepsis and its aggressive management in neonates $[10,11]$.

The evaluation of neonatal sepsis is important and there is an urgent need to know whether the baby has sepsis so as to institute treatment as quickly as possible. Hence this study is undertaken to determine the incidence of early onset neonatal sepsis in relation to PROM of more than 18 hours.

\section{Objectives}

To determine the incidence of neonatal sepsis in relation to prolonged rupture of membranes (PROM) $>18$ hours and associated risk factors for early onset neonatal sepsis (EONS) in neonates born to mothers with history of PROM.

\section{Material and Methods}

The present prospective study was conducted from December 2013 to November 2014 in GSL medical college and hospital, Rajahmundry. All the neonates born to mothers with history of prolonged rupture of membranes $>18$ hours during study period was formed sample size for present study. Institutional Ethical Clearance was obtained prior begin of the study. The purpose of study was explained and Informed consent was obtained from mothers.

\section{Selection criteria}

Inclusion criteria: All neonates born to mothers with Prolonged rupture of membranes more than 18 hours in GSL medical college and hospital, Rajahmundry.

\section{Exclusion criteria}

1. Antepartum hemorrhage

2. Toxemia of pregnancy

3. Major medical illness to the mother other than infections

4. Mothers with PROM $<18$ hours

5. Neonates with major congenital malformations

6. Neonates presenting with symptoms after 72 hours after birth

7. Neonates who underwent any mode of resuscitation other than routine care.

Data collection procedure: A detailed history was taken including age, parity, Obstetric history of the mother with emphasis on exact time of rupture of membranes, duration history and antibiotics before labour were evaluated.

Detailed birth history including resuscitation details, Apgar score and gestational age assessment were evaluated. In examination of the neonate the pulse, respiratory rate, CFT and temperature were noted followed by systemic examination. Required investigations are done and followed during their hospital stay.

Diagnostic criteria- Diagnosis of neonatal sepsis made in neonates having clinical signs and symptoms consistent with sepsis having any two parameters of sepsis screen positive or neonate with positive blood culture.

Pediatric Review: International Journal of Pediatric Research Available online at: www.medresearch.in 445|P a g e 
Original Research Article

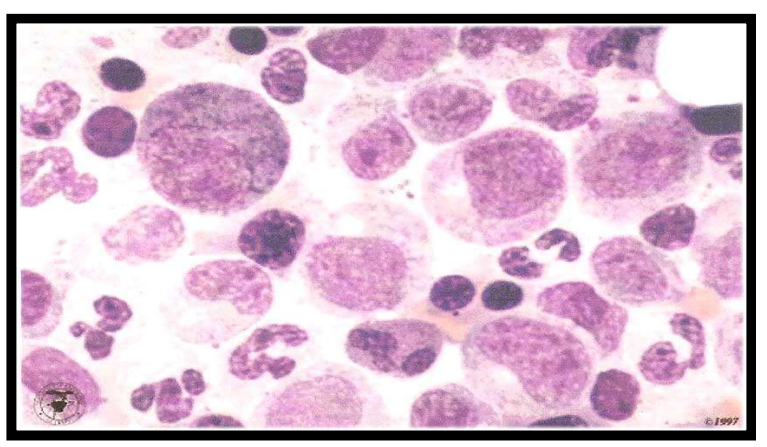

Figure-1: Band Neutrophil

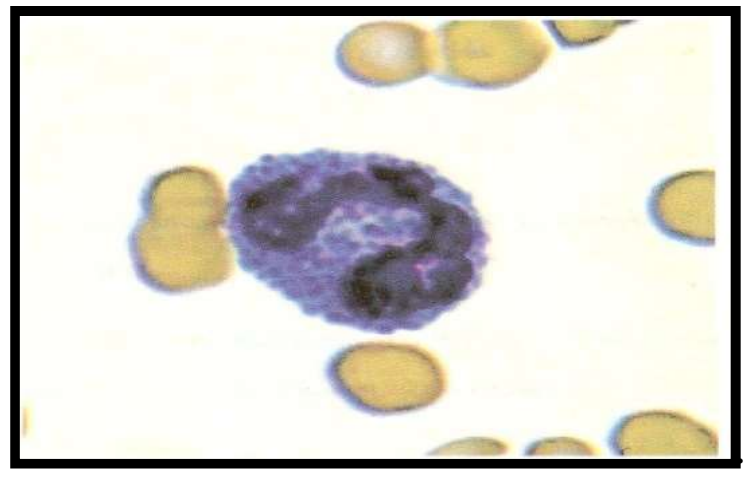

Figure-2: Toxic granules

\section{Avitex CRP}

\section{Avitex - CRP latex test}

Avitex - CRP is a rapid latex agglutination test for the detection of $\mathrm{C}$ - reactive protein. In human serum when the latex suspension is mixed with serum containing elevated CRP levels on a slide clear agglutination is seen within 2 minutes.

A positive result is indicated by the obvious agglutination of the latex, in a clear solution. A negative result is indicated by no change in the latex suspension on the test slide. Positive result will be obtained at a serum CRP concentration above $6 \mathrm{mg} /$ liter and negative result will be obtained at $6 \mathrm{mg} /$ liter and below.

Blood culture- Blood was drawn before starting any antimicrobial agent with aseptic precaution from a peripheral vein. The volume of blood drawn was $2 \mathrm{ml}$ and transferred to a glucose broth in a dilution of $1: 10$. This was then sent to department of Microbiology for culture and sensitivity. The bacteria were identified by the morphological, biochemical and serological reactions.

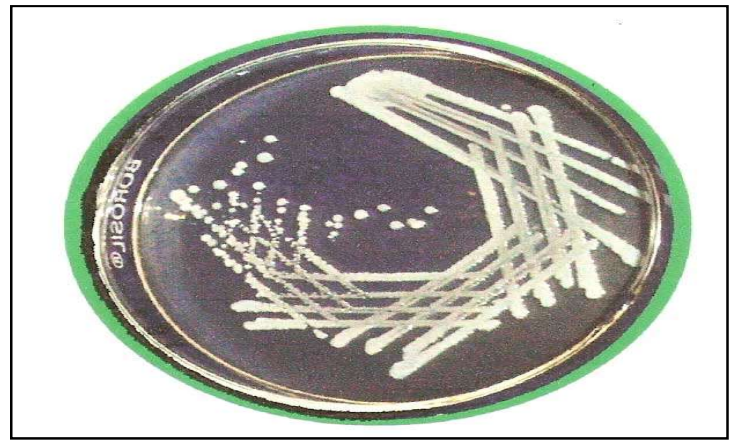

Figure-3: Growth of staphylococcus aures. 


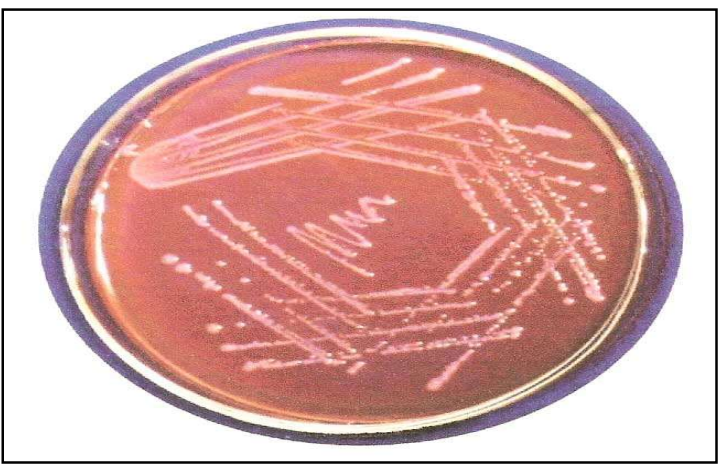

Figure - 4: Growth of Klebsiella.

Statistical analysis: Descriptive statistics such as mean, SD and percentage was used to present the data. Further, sensitivity, specificity, positive predictive value, negative predictive value was calculated. Data analysis was performed by using Microsoft excel and SPSS v20.0.

\section{Results}

Out of 200 neonates, 60 neonates had clinical signs and symptoms consistent with sepsis and are included in the category "Clinical sepsis"

Out of 200 neonates 29 neonates had confirmed sepsis i.e. clinical features + either sepsis screen and/or blood culture positive and are included in the category "Confirmed sepsis"

Table-1: Distribution of cases according to clinical sepsis and confirmed sepsis.

\begin{tabular}{|l|c|c|c|}
\hline \multicolumn{1}{|c|}{ Variable } & Total cases & Clinical sepsis & Confirmed sepsis \\
\hline Sex & & & $18(62 \%)$ \\
\hline Male & 101 & $38(63.33 \%)$ & $11(38 \%)$ \\
\hline Female & 99 & $22(36.66 \%)$ & $19(66 \%)$ \\
\hline Birth weight (gms) & & & $10(34 \%)$ \\
\hline$<2500$ & 86 & $41(68.33 \%)$ & $18(61.5 \%)$ \\
\hline$>2500$ & 114 & $19(31.67 \%)$ & $11(38.5 \%)$ \\
\hline Gestational age & & & $16(68.5 \%)$ \\
\hline$<37$ weeks & 81 & $35(58.33 \%)$ & $13(31.5 \%)$ \\
\hline$>37$ weeks & 119 & $25(41.67 \%)$ & \\
\hline Mode of delivery & & & $14(48.27 \%)$ \\
\hline Normal vaginal delivery & 121 & $43(71 \%)$ & $15(51.72 \%)$ \\
\hline Caesarean section & 79 & $17(28 \%)$ & \\
\hline Duration of PROM & & & 26 \\
\hline $18-24$ hours & 116 & 34 & \\
\hline $24-72$ hours & 84 & & \\
\hline
\end{tabular}

In the present study it was observed male neonates with confirmed sepsis are more $62 \%$ compared to female neonates $38 \%$. Incidence of sepsis is higher in low birth weight neonates (66\%) compared to normal birth weight babies (34\%). Neonatal sepsis had a higher rate of incidence in preterm neonates $(61.5 \%)$ than in term neonates $(38.5 \%)$. Incidence of sepsis was found to be higher in neonates born by normal vaginal $(68.5 \%)$ delivery compared to cesarean section (31.5\%). There is no much difference between incidence of sepsis and duration of PROM, as the present study takes only 18 hours to 72 hours duration of PROM into consideration. PROM above 72 hours not included in the present study as it causes late onset sepsis (Table-1). 


\section{Original Research Article}

Table-2: Distribution of cases in relation to sensitivity patterns of various sepsis screen parameters

\begin{tabular}{|c|c|c|}
\hline Variable & No. of cases & Percentage \\
\hline Low WBC count & 18 & 9.00 \\
\hline Neutropenia & 19 & 9.50 \\
\hline IT ratio & 17 & 8.50 \\
\hline Micro ESR & 20 & 10.0 \\
\hline CRP & 26 & 13.0 \\
\hline
\end{tabular}

Out of all the five sepsis screen parameters CRP has the highest sensitivity $(90.90 \%)$, with 26 cases showing positive CRP in 29 cases of confirmed sepsis followed by Micro ESR (65.51\%) with 19 cases positive in 29 cases of confirmed sepsis (Table-2).

Table-3: Distribution of cases in relation between Sepsis screen and Blood culture

\begin{tabular}{|l|c|c|c|c|}
\hline Parameters & Culture positive & Percentage & Culture negative & Percentage \\
\hline Total WBC count & 13 & 59.09 & 5 & 50.0 \\
\hline Absolute neutrophil count & 14 & 63.63 & 5 & 33.33 \\
\hline Band count & 13 & 59.09 & 4 & 30.76 \\
\hline Micro ESR & 16 & 72.72 & 4 & 30.76 \\
\hline CRP & 20 & 90.90 & 6 & 75.0 \\
\hline
\end{tabular}

Total number of neonates with clinical sepsis was 60 . Blood culture is done on all the 60 neonates with clinical signs and symptoms. Out of 60, 22 neonates are positive for blood culture. Number of neonates with confirmed sepsis i.e- either 2 parameters of sepsis screen positive and/or blood culture positive was 29 (Table-3).

Table-4: Sepsis screen parameters.

\begin{tabular}{|l|c|c|c|c|}
\hline Parameters & Sensitivity & Specificity & $\begin{array}{c}\text { Positive } \\
\text { predictive value }\end{array}$ & $\begin{array}{c}\text { Negative } \\
\text { predictive value }\end{array}$ \\
\hline Total WBC count & $59.09 \%$ & $71.42 \%$ & $86.66 \%$ & $50.0 \%$ \\
\hline Absolute neutrophil count & $63.63 \%$ & $72.34 \%$ & $85.30 \%$ & $33.33 \%$ \\
\hline Band count & $59.09 \%$ & $57.14 \%$ & $81.25 \%$ & $30.76 \%$ \\
\hline Micro ESR & $72.72 \%$ & $56.03 \%$ & $84.21 \%$ & $31.62 \%$ \\
\hline CRP & $90.90 \%$ & $85.71 \%$ & $95.23 \%$ & $75.0 \%$ \\
\hline
\end{tabular}

Out of the five sepsis screen parameters, CRP has the highest sensitivity and specificity rates (Table-4).

Table-5: Bacterial pathogens isolated in blood culture of neonates.

\begin{tabular}{|l|c|c|}
\hline Bacterial isolate & No. of cases & Percentage \\
\hline Staphylococcus aureus & 10 & 45.45 \\
\hline Coagulase negative staph aureus & 6 & 27.27 \\
\hline Klebsiella & 3 & 13.6 \\
\hline E.coli & 3 & 13.6 \\
\hline
\end{tabular}

Most common bacterial isolate in the present study was Staphylococcus aureus (45.45\%) followed by Coagulase negative staph aureus (27.27\%), Klebsiella (13.6\%) and E.coli (13.6\%) (Table-5).

Pediatric Review: International Journal of Pediatric Research Available online at: www.medresearch.in 448|P a g e 


\section{Discussion}

Sex distribution: In present study male babies have more incidence of early onset neonatal sepsis compared to female babies, which is consistent with Hitesh $\mathrm{J}$ Assudani study [12]. Aletayeb Setaland Celiciac C et al study also observed incidence of sepsis is more in male babies compared to female babies $[13,14]$.

In Betty Chacko and Inderpreet Sohi study incidence of neonatal sepsis was similar to male and female neonates which is not consistent with the present study [15]. There was significant difference in the incidence of sepsis between male neonates (62\%) and female neonates $(38 \%)$ ( $\mathrm{p}$ value $<0.05)$.

Bias for male sex, place of study, sample including other factors may be responsible for increased number of male cases in these studies.

Birth weight: In present study there was statistical significance in the incidence of neonatal sepsis was more in low birth weight neonates <2500gm $(66.1 \%)$ compared to normal birth weight neonates (33.9 5) (p value $<0.05$ ).

Similar results were obtained in other studies such as Yasmeen $\mathrm{J}$ et al., $76.3 \%$ in LBW neonates vs $23.7 \%$ in normal birth weight neonates [16] and Rajashri Basu Syamal Kumar study $(74.87 \%$ vs $23.7 \%)$ [17]. However, in Woranart et al study, incidence of neonatal sepsis was almost similar in both low birth weight and normal birth weight neonates [18].

LBW babies are mostly also premature and are predisposed to sepsis due to multiple reasons like immune incompetence at various levels of defense, more subjected to invasive interventions etc.

Gestational age: In present study there was significant difference in preterm neonates $(61.5 \%)$ compared to term babies $(38.5 \%$ ) ( $\mathrm{p}$ value $<0.05$ ). Kifah Al-Q Fatin -Al awayshah study had similar results as the present study $61.5 \%$ in preterm babies compared to $38 \%$ in term babies [11].

However, Woranart et al study was not consistent with the present study, $42.3 \%$ in preterm babies compared to $57.69 \%$ in term babies [18].
Due to immature immune function, premature babies are more prone to infections compared to term.

Mode of delivery: In present study there was significant difference in the incidence of neonatal sepsis in neonates born by normal vaginal delivery $(68.5 \%)$ vs caesarean section $(31.5 \%$ ) (p value $<0.05)$.

Other studies which are consistent with the present study are Kifah Al-Q Fatin -Al awayshah study, in which incidence of neonatal sepsis in neonates born by NVD are (54\%) compared to neonates born by caesarean section $(20 \%)$ [11].

Sanyal Mukherjee study and Kodkany, Telang study showed much greater difference in the incidence of neonatal sepsis in neoantes born by normal vaginal delivery compared to caesarean section ( $87 \%$ vs $13 \%)$ and $(81 \%$ vs $19 \%)$ respectively $[19,20]$.

Duration of PROM: In present study percentage of cases with sepsis during 18 to 24 hours study period is slightly less than 24 to 72 hours, but there was no significant difference between incidence of sepsis between 18 to 24 hours and 24 to 72 hours (p value $>0.05$ ).

In Woranart et al study percentage of cases with sepsis during 18 to 24 hours period is more compared to 24 to 72 hours period which is not consistent with present study [18].

As present study included neonates born to mothers with PROM only between 18 to 72 hours period, results may not be significant to study the incidence of neonatal sepsis in relation to PROM.

Presenting symptom: In present study Respiratory distress (54.5\%) was the commonest presenting symptom followed by refusal of feeds $(47.5 \%)$ and lethargy $(43.5 \%)$.

In Jain N K et al study also, respiratory distress (42.6\%) was the commonest presenting symptom [21]. However, in Omer et al study, fever $(63.33 \%)$ was the commonest presenting sign followed by respiratory distress [22]. Earliest clinical features of neonatal sepsis

Pediatric Review: International Journal of Pediatric Research Available online at: www.medresearch.in 449|P a g e 


\section{Original Research Article}

are often subtle and nonspecific therefore a high index of suspicion is needed for early diagnosis specially so if risk factors are also present clinical features and further course in neonatal sepsis depends on various factors like birth weight, place of delivery, age of newborn, intervention in preventable factors for sepsis, availability, accessibility, affordability and timely referral of baby to an appropriate center. Therefore, variation in different parameters may be observed in various studies.

H/o pre-natal antibiotic intake: In present study the percentage of neonatal sepsis is higher in mothers with PROM who did not receive prenatal antibiotics $(20.65 \%)$ compared to mothers who had prenatal antibiotic intake $(9.25 \%)$.

Similar results were obtained by Kifah Al-Q Fatin -Al awayshah study, the percentage of neonatal sepsis is only $4.4 \%$ in neonates born to mothers who received prenatal antibiotics compared to $11 \%$ neonates whose mothers did not receive prenatal antibiotics for PROM [11]. Preterm premature rupture of fetal membranes defined as rupture before 37 weeks. Occur in $2-4 \%$ of pregnancies, is associated with intrauterine infection and increased risk of neonatal sepsis.

There are several studies in which women presenting with preterm PROM without labor were randomized to receive an antibiotic or placebo. Overall, these studies have shown that antibiotics prolonged the latency period until delivery, reduced maternal choriomnionitis and reduced neonatal complications, including respiratory distress syndrome, IVH, pneumonia and sepsis as in present study newborns whose mothers received antibiotic before have good outcome. Antibiotic have become an important part of the recent advances in the treatment of PROM. Many studies have demonstrated that antibiotic therapy prolong pregnancy latency time and decreases maternal and neonatal morbidity.

Sensitivity and specificity of absolute neutrophil count: Leucocyte count varies from 8000 to $20,000 /$ cumm during the first 28 days of life without demonstrable disease. Neonatal sepsis is usually associated with leucopenia $<5000 \mathrm{cu} / \mathrm{cumm}$. f This finding was taken along with other parameters for early diagnosis of neonatal sepsis with reasonable sensitivity and specificity.
Absolute neutrophil count of less than 1500cell/cumm is taken as cut off value in cases of neonatal sepsis. The sensitivity and specificity of absolute neutrophil count in present study is $63.63 \%$ and $71.42 \%$ respectively. This is similar to sensitivity of S. Khurshid Anwer, Sultan Mustafa study where sensitivity is about $61.9 \%$ which is almost similar to the present study, but specificity is lower around $51.72 \%$ compared to present study with specificity of about $71.42 \%$ [23].

In Ahmed Elewa et al study [24] and B. Venkatarao, G. Israel, P. Kamala study [25] specificity is almost similar to the present study which is $74 \%$ and $73.3 \%$ respectively whereas specificity of present study is $71.42 \%$.

Sensitivity and specificity of immature to total neutrophil ratio: Band cell count to total neutrophil count ratio (I/T ratio): $\geq 0.2 \mathrm{I} / \mathrm{T}$ ratio $=$ no. of band cells / no. of neutrophils.

The immature polymorph nuclear leucocytes, (stab cells) characterized by further condensation of nuclear chromatin and transformation of nuclear shapes into sausage and band forms with more or less uniform diameters throughout their length, measuring 10- $16 \mu \mathrm{m}$ and the nucleus centrally or eccentrically placed with light purplish blue. Cells without their complete formation of distinct lobes (usually connected by a filamentous strand) are classified as band forms.

Juvenile/band forms $10-16 \mu \mathrm{m}$ size nucleus is centrally or eccentrically placed and band shape of uniform thickness, light purplish blue. They normally constitute $<6 \%$ neutrophils.

Sensitivity and specificity of CRP: The sensitivity and specificity of CRP in present study is higher with $90.90 \%$ and $85.71 \%$ respectively. The present study is consistent with Chauhan $\mathrm{S}$ et al study with a sensitivity and specificity of $92.30 \%$ and $85.71 \%$ [26].

But in Boma A West et al study sensitivity and specificity are lower with $74 \%$ which is not consistent with the present study [27]. CRP passes the placenta only in very low quantities; therefore, any elevation in the neonate always represents endogenous synthesis. De novo hepatic synthesis starts very rapidly after a single stimulus with serum concentrations rising above $5 \mathrm{mg} / 1$ by about 6 hours and peaking around 48 hours.

Pediatric Review: International Journal of Pediatric Research Available online at: www.medresearch.in 450|P a g e 


\section{Original Research Article}

In diagnosis of early onset sepsis previous studies reported on widely differing sensitivities and specificities of CRP ranging from $29 \%$ to $100 \%$ and from $6 \%$ to $100 \%$, respectively. These extreme variations are a result of different reference-values, test methodologies, patient characteristics and inclusion criteria, number of samples taken, and sampling time. Furthermore, definitions of sepsis widely differ between studies making serious comparisons hardly possible.

On the other hand, a raised CRP is not necessarily diagnostic for sepsis, as elevations may as well occur due to the physiologic rise after birth or non-infection associated conditions. Therefore, concerns were raised about the reliability of CRP during the early stage of the disease being neither able to diagnose nor to rule out an infection with certainty.

Positive blood culture: Blood culture was done in all the 60 neonates with clinical sepsis. Out of 60,22 neonates had positive blood culture with percentage positivity of about $36.66 \%$.

Compared to other studies, blood culture positivity is slightly lower with $36.66 \%$ in the present study. In Kayange et al study, positive blood culture was seen in 57 among 121 clinical sepsis cases [29], and in Eman M. Rabieshehab el din et al study blood culture positivity was seen in nearly $40.7 \%$ cases with $140 / 344$ clinical sepsis cases [29].

Low blood culture positivity in the present study may be due to small sample size compared to other two studies. However, this rate is comparable to rates reported in other developing African and Asian countries as A. S. Ahmed, M. A. Chowdhury et al study Bangladesh (34.88\%) and J. Mugalu, M. K. Nakakeeto, et al study, Uganda (37\%) [30, 31].

Incidence of early onset neonatal sepsis among PROM mothers: Incidence of EONS in present study is $14.5 \%$ which is slightly lower compared to other studies. Nili AA, Shams Ansari study shows 18.5\% which is slightly above the present study [32].

Ratanakorn $\mathrm{W}$ et al study shows the highest incidence about $27.9 \%$ [18]. These differences are probably due to use of different criteria for diagnosis of neonatal sepsis and/or inclusion of coagulase negative staphylococcus and other contaminants.
Isolates in early onset neonatal sepsis: In present study Staphylococcus aureus was the commonest isolate $(45.45 \%)$ followed by CONS $(27.27 \%)$. This was contrary to NNPD-2002-1003 where klebsiella was the commonest isolate followed by staph aureus [33]. In another study conducted by Shashikala. S. Thallur et al on 242 babies klebsiella was the commonest isolate [34]. E.coli was the commonest isolate in a study done by Kerur Basavaraju et al study [35] Staph aureus was the commonest pathogen (48.7\%) followed by coliforms in study conducted by university of Calabar, Nigeria 2005 by Martin. M. Meremikwu and Chuckwumek study [36].

Limitations of the present study: Further study in large number of patients is required to comment regarding incidence rates, complications and associated risk factors for early onset neonatal sepsis.

\section{Conclusion}

The evaluation of neonatal sepsis is important and there is an urgent need to know whether the baby has sepsis so as to institute treatment as quickly as possible.

\section{What the study adds to the existing knowledge?}

Literature on incidence of early onset neonatal sepsis has shown different rates which make a meaningful addition to the existing literature by conducting the present study for timely recognition and understanding of common issues related to PROM of more than 18 hours.

\section{Author's contribution}

Dr. G Kalyan Chakravarthi: Study design and manuscript preparation.

Dr. S Surya Veera Kumar: Study design and manuscript preparation

Funding: Nil, Conflict of interest: None initiated, Permission from IRB: Yes

\section{Reference}

1.Shahraki AD, Khan KS, Wojdyla D, Say L, Gulmezoglu AM, Van Look PF, Naghavi M, Rutstein DD, Berenberg W, Chalmers TC, Child CG. World Health Report: Make Every Mother and Child Count. J Med Sci.2005;7(8):1066-1074.doi: 10.3923/jms.2007. 1340. 1344

Pediatric Review: International Journal of Pediatric Research Available online at: www.medresearch.in 451|P a g e 


\section{Original Research Article}

2.World Health Report: Level and trends in child mortality. Geneva: WHO 2011. Available from https:// www. unicef. org >files > Child_Mortality_Report_ 2011_Final

3.Black RE, Cousens S, Johnson HL, Lawn JE, Rudan I, Bassani DG, et al. Global, regional, and national causes of child mortality in 2008: a systematic analysis. Lancet. 2010;375(9730): 1969-1987. doi: 10.1016/ S0140-6736(10)60549-1. Epub 2010 May 11.

4. Mahmood A, Fazal ur Rehman, Chughtai F. A survey of infection control practices in the delivery room and nursery to investigate and control the high rate of neonatal sepsis: an experience at a secondary care hospital. J Pak Med Assoc. 2008;58(5):237-240.

5. Popowski T, Goffinet F, Maillard F, Schmitz T, Leroy S, Kayem G. Maternal markers for detecting early-onset neonatal infection and chorioamnionitis in cases of premature rupture of membranes at or after 34 weeks of gestation: a two-center prospective study. BMC Pregnancy Childbirth. 2011;11:26. doi: 10.1186/ 1471-2393-11-26.

6. Kilbride HW, Thibeault DW. Neonatal complications of preterm premature rupture of membranes. Pathophysiology and management. Clin Perinatol. 2001;28(4):761-785. doi:https://doi.org/10.1016/S00955108(03)00076-9

7. Mercer BM, Goldenberg RL, Das AF, Thurnau GR, Bendon RW, Miodovnik M, Ramsey RD, Rabello YA. What we have learned regarding antibiotic therapy for the reduction of infant morbidity after preterm premature rupture of the membranes. InSeminars in Perinatology. WB Saunders. 2003 Jun 1 (Vol. 27, No. 3, pp. 217-230).

8. Medina TM, Hill DA. Preterm premature rupture of membranes: diagnosis and management. Am Fam Physician. 2006;73(4):659-664.

9. Boskabadi H, Maamouri G, Mafinejad S. Neonatal complications related with prolonged rupture of membranes. Maced J Med Sci. 2011;4(1):93-98. doi: 10.3889/MJMS.1857-5773.2011.0159

10. Mercer B. Antibiotics in the management of PROM and preterm labor. Obstet Gynecol Clin North Am. 2012;39(1):65-76. doi: 10.1016/j.ogc.2011.12.007. Epub 2012 Jan 28.
11. Al-QaQa K, Al-Awaysheh F. Neonatal outcome and prenatal antibiotic treatment in premature rupture of membranes. Pakistan J Med Sci.2005;21(4):441-444.

12. Assudani HJ, Gusani JK, Mehta SJ, Agravat HH, Kothari K. Bacteriological profiles of septicaemia in neonates at tertiary care hospital, Gujarat, India. J Res Med Dent Sci. 2015;3(2):148-151. doi: 10.5455/ jrmds. 20153212

13.Aletayeb SM, Khosravi AD, Dehdashtian M, Kompani F, Aramesh MR. Identification of bacterial agents and antimicrobial susceptibility of neonatal sepsis: A 54-month study in a tertiary hospital. Af J Microbiol Res. 2011;5(5):528-531. doi: 10.1186/14712334-13-89

14. Cecilia CM, Mary AC, Elizabeth EG, Jonathan GL, Joanne JL et al. Etiology of neonatal sepsis in five urban hospitals in the Philippines. PIDSP Journal. 2011; 12:75-85.

15. Chacko B, Sohi I. Early onset neonatal sepsis. Indian J Pediatr. 2005;72(1):23-26. doi:10.1007/ bf 02760574

16. Yasmeen J, Al-Bayaa, NedhalS.Ayoub, Sevan Najem Alwan. Relationship between Neonatal septicemia and birth weight. Fac Med Baghdad. 2012; 54 (2): 151-153.

17. Rajarshi B, Syamalkumar B. Study on Correlation between Sepsis Screening and Blood Culture in Neonatal Sepsis. IOSR J Dent Med Sci. 2014;13(5): 52-56.

18. Ratanakorn W, Srijariya W, Chamnanvanakij S, Saengaroon P. Incidence of neonatal infection in newborn infants with maternal history of premature rupture of membranes (PROM) for 18 hours or longer by using pharmong Kutklar Hospital clinical practice guidelines (CPG) J Med Assoc. Thai. 2005; 8(7): 973-978

19. Sanyal MK and Mukherjee TN. Premature rupture of membrane; an assessment from a rural medical college of West Bengal; J. Obstet Gynecol India. 1990; 40 (4): 623-628.

20.Kodkany BS, Telang MA. Premature rupture of membranes. A study of 100 cases. J. Obstet Gynecol India. 1991;41(4):492-496.

Pediatric Review: International Journal of Pediatric Research Available online at: www.medresearch.in 452|P a g e 


\section{Original Research Article}

21. Jain NK, Jain VM, Maheshwari S. Clinical profile of neonatal sepsis. Kathmandu Univ Med J (KUMJ). 2003; 1(2):117-20.

22. Magzoub OS, Ahmed MA, Abdelgadir YS. Clinical presentation of neonatal sepsis in paediatric ward at Khartoum North Teaching Hospital, Sudan. Basic Research Journal of Medicine and Clinical Sciences 2015; 4(4):116-120.

23. Anwer SK, Mustafa S. Rapid identification of neonatal sepsis. J -Pak Med Assoc. 2000; 50 (3): 94-7.

24. Elewa A, Anber N, El Sayed ZM, Basem SA. El Deek. Evaluation of Microbiologic and Hematologic Parameters and E-Selectin as Early Predictors for Outcome of Neonatal Sepsis. Int J Curr Microbiol App Sci. 2015;4(11):665-673.

25. Venkatarao B, Israel G, Kamal P. Diagnostic Value of C- Reactive Protein and Hematological Parameters in Neonatal Sepsis. IOSR-JDMS. 2015;14(4):30-38.

26. Chauhan SB, Vaghasia V, Chauhan BB. C-Reactive Protein (CRP) in Early Diagnosis of Neonatal Septicemia. Nat J Med Res. 2012;2(3):276-278.

27. West BA, Peterside O, Ugwu RO, Eneh AU. Prospective evaluation of the usefulness of C-reactive protein in the diagnosis of neonatal sepsis in a subSaharan African region. Antimicrobial resistance and infection control. 2012;1(1):22. doi: 10.1186/20472994-1-22

28. Kayange N, Kamugisha E, Mwizamholya DL, Jeremiah S, Mshana SE. Predictors of positive blood culture and deaths among neonates with suspected neonatal sepsis in a tertiary hospital, Mwanza-Tanzania BMC Pediat. 2010;10(1):39. doi: 10.1186/1471-243110-39.
29. Eman M. RabieShehab El-Din, Mohamed M. Adel El-Sokkary, Mohamed Reda Bassiouny, Ramadan Hassan. Epidemiology of Neonatal Sepsis and Implicated Pathogens: A Study from Egypt. Bio Med Res Inter07/2015. 2015:1-1. doi: http://dx.doi.org/ 10. $1155 / 2015 / 509484$

30. Ahmed AS, Chowdhury MA, Hoque M, Darmstadt GL. Clinical and bacteriological profile of neonatal septicemia in a tertiary level pediatric hospital in Bangladesh. Indian Pediat. 2002;39(11):1034-1039.

31. Mugalu J, Nakakeeto MK, Kiguli S, Kaddu Mulindwa DH. Aetiology, risk factors and immediate outcome of bacteriologically confirmed neonatal septicaemia in Mulago hospital, Uganda. African Health Sciences. 2006;6(2):120-126.

32. F-Nilli AA, Shams A. Neonatal Complications of premature rupture of membrane. Acta Medica Iranica. 2003; 41(3):175-179.

33. Report of the National Neonatal Perinatal Database. Report 2002-2003. NNPD Network. [Last accessed on 2013 Dec 17]. Available from: http://www.newborn whocc. org/pdf/nnpd_report_2002-03.PDF .

34.Shashikala S.TallurA.V. Kasturi,Shobha D.Nadgir,B V. S. Krishna. Clinico-bacteriological study of neonatal septicemia in Hubli. Ind J Pediat. 2000;67 (3): 169-174.

35. Kerur BM, Bhat BV, Harish BN, Habeebullah S., Kumar CU. Maternal Genital Bacteria and Surface Colonization in Early Neonatal Sepsis. Indian J Pediatr 2006; 73(1):29-32. doi: https://doi.org/10.1007/BF027 58256

36. Meremikwu MM, Nwachukwu CE, Asuquo AE, Okebe JU, Utsalo SJ. Bacterial isolates from blood cultures of children with suspected septicaemia in Calabar, Nigeria. BMC infectious diseases. 2005;5(1):110

\section{How to cite this article?}

Chakravarthi G.K, S. Surya Veera Kumar. Incidence of neonatal sepsis in relation to prolonged rupture of membranes (PROM) $>18$ hours and associated risk factors for early onset neonatal sepsis (EONS). Int J Pediatr Res.2019;6(09): 444-453.doi:10.17511/ijpr. 2019.i09.02

Pediatric Review: International Journal of Pediatric Research Available online at: www.medresearch.in 453|P a g e 